\title{
Cultural Issues in Anisul Hoque's Ayeshamangal and its Translation
}

Tanmoy Mazumder (Corresponding author)

Department of English, Jashore University of Science and Technology, Bangladesh

Email: tanmoy_eng@just.edu.bd

Received: $27 / 06 / 2020$

Accepted: 12/08/2020

Published: 01/09/2020

Volume: 1 Issue: 3

How to cite this paper: Mazumder, T. (2020). Cultural Issues in Ayeshamangal and its Translation. Journal of Critical Studies in Language and Literature, 1(3), 64-69

DOI: https://doi.org/10.46809/jcsll.v1i3.29

Copyright ( 2020 by author(s) and Global Talent Academy Ltd. This work is licensed under the Creative Commons Attribution International License (CC BY 4.0).

http://creativecommons.org/licenses/by/4.0/

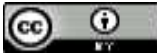

\begin{abstract}
Anisul Hoque's Ayeshamangal (2010) has been a text of quite critical acclamation in Bangladesh due to its presentation of a courageous village woman, Ayesha- who struggles in the context of post-independent political turmoil in Bangladesh in synchronization of a legendary figure in Bengali folk myth, Behula. This study provides a reading of Ayeshamangal's English translation, The Ballad of Ayesha (2018), in order to find out the cultural implications in the source text (ST) and the target text (TT). Translators face the problem of treating the cultural aspects of a ST and then its appropriate transfer to the TT and target language (TL). In this novel, the translator faces such a dilemma on various occasions. The ST extensively uses a certain dialect of the source language (SL). This paper investigates the transfer of this dialect of Rangpur region to the target language (TL). The paper examines the techniques used by the translator to successfully convey the aspects of Bengali culture into the TL of the novel. This study also focuses on translations of certain lexical contents, ways of life and the myth of Behula. Thus, the current paper investigates the translation strategies and procedures used in the TT from certain theoretical perspectives in translation studies and argues that the translator attempts for an overall equivalent effect in the TT, though he fails to provide the taste of important cultural aspects of Bangladesh to the TT readers.
\end{abstract}

Keywords: Adaptation, Borrowing, Calque, Cultural Implication, Ecology, Equivalence, Literal Translation, Myth of Behula

\section{Introduction}

The idea of culture is fundamental and inseparable to any approach to translation in any language all over the world. Now, if we define translation as "the process of changing something that is written or spoken into another language" (Oxford learner's dictionary) and as a text production generated by a source text, translation of a text from its source language (SL) into a foreign language (the target language) can be regarded as an instance of intercultural communication. Again, for Toury (2004), "translation is a kind of activity which inevitably involves at least two languages and two cultural traditions." (p. 207). So, it is obvious that the translators always face the problem of treating the cultural aspects implied in a source text (ST) and then, finding out the best possible solution to convey them in the target language (TL). And, for Nida (2004), "a translation may involve not only difference of linguistic affiliation but also highly diverse culture..." (p.157) and these is sues may differ in scope depending on the cultural and linguistic distance between the languages concerned. The cultural suggestions for translation might concern various issues including lexical contents, syntax, history, certain mythologies, ideologies and ways of life in a concerned culture of the source text (ST). So, a translator must take into account different possibilities and then decide on the best method which seems appropriate in each specific case. Before that, the 
translator should have a clear idea about the aim and purpose of the translation and also, about his ideal target reader. The translation should make sense and in addition, it "must also convey the 'spirit and manner' of the original." (Nida, 2004, p. 159).

The present study investigates cultural issues in Ayeshamangal and its translation The Ballad of Ayesha. Ayeshamangal, a Bengali novel, has been quite a critic acclaimed novel from its publication in 2010. The Ballad of Ayesha, published in 2018 by HarperCollins, is an English translation of Ayeshamangal by Inam Ahmed. Translating the Ayeshamangal in English is surely a complex issue as the novel deals with diverse cultural issues in Bangladesh. The novel sets out in two different regions in Bangladesh- Rangpur and Dhaka, captures complex historical events in Bangladesh from 1975 to 1997, invokes a popular mythological story of Behula in parallel to the tragedy in life of the protagonist in the novel, and deals with the issue of blending all these things along with all the necessary cultural aspects in a single narrative. This study attempts to investigate all these issues in The Ballad of Ayesha. The paper would examine how the translator deals with the cultural issues present in the source text (ST) that represents rural regions of Rangpur, unique in its distinct social and cultural practices; the paper also attempts to study the translator's strategy to deal with the Rangpur dialect of Bengali language obvious in the source text (ST), the issues of transferring cultural aspects of the source culture in the target text (TT), the translation strategy in dealing with different lexical contents, the representation of the mythological story of Behula and in the end, the translator's attempt for equivalence. Thus, this study, with a view to unveiling the translation approach, firstly, would discuss the nature of the source text (ST), the ideal source text reader and the ideal target text (TT) reader and then, the translation strategies and procedures would be analyzed.

\section{The Nature of Ayeshamangal and the Readers}

\subsection{The Ideal ST Reader}

Ayeshamangal is a tale of a personal tragedy of the protagonist of the novel, Ayesha, a widowed woman, wife of an executed soldier in Bangladesh Air Force. The novel narrates the history of an uprising in Bangladesh Air Force in 02 October1977 and then, blends the personal tragedy of an individual with a national tragedy in a parallel reference to a popular mythological story in Bengal of a woman of indomitable spirit named Behula, found in Manashamangal, a thirteenth or fourteenth century epic in Bengali literature. Tragedy of Ayesha in Ayeshamangal is compared to the tragedy of Behula who lost her husband Lakhinder due to the wrath of the goddess Manasha. The novel sets out in Rangpur and Dhaka regions. The Rangpur dialect and Dhaka-centric colloquial language along with all the social norms, cultural aspects and ideological point of views build up this novel as an interesting one for the readers. Now, the question that we need to ask is that for whom Ayeshamangal has been written and who the ideal source text readers are. And, then we may decide whether the ideal ST readership here corresponds to the potential TT reader. Here, the subject matter of the original text is literary in nature it blends history, mythology and local culture. Regarding this point, Kate James (2002) in her article titled, "Cultural Implications for Translation", points out:

Coulthard (1992) highlights the importance of defining the ideal reader for whom the author 'attributes knowledge of certain facts, memory of certain experiences ... plus certain opinions, preferences and prejudices and a certain level of linguistic competence.' When considering such aspects, it should not be forgotten that the extent to which the author may be influenced by such notions is dependent on his own sense of belonging to a specific socio-cultural group. (para. 12).

Hoque, the author of Ayeshamangal, belongs to the Rangpur region. The author supposes that his ideal reader has knowledge of historical events of post 1971 Bangladesh and Manashamangal, the epic in Bengali folklore. Again, memory of certain experiences among the readers seems to be a consideration of the author. The memories of a tumultuous time in the post-liberation Bangladesh, especially of 1977 and familiarity with Rangpur dialect are assumed to be common in the ideal reader. Next, the source text seems to be asking for a readership that contains certain opinions, preferences and ideological stand associating the historical events concerning the killing of Bangabandhu Sheikh Mujibur Rahman in 1975; the betrayer Khandokar Moshtaq; Ziaur Rahman's becoming the ruler of Bangladesh; the uprising of 1977 etc. Finally, Ayeshamangal may be considered a novel intended for an educated, middle-class readership.

\subsection{The Ideal TT Reader}

Having a clear knowledge of the possible readers of the translation, and understanding their expectations and competence level of assuming the cultural context of the original text is an important thing to be considered by translators. If the target text readers are not understood well, then, there is a possibility of an unorganized and uncommunicative translation (in the sense that the translator will be without focus and the TT readers will fail to apprehend the ST issues often). So, after determining the source text readership, translators should make considerations regarding the ideal target text reader. We can point out to Coulthard's (1992) observation concerning this:

The translator's first and major difficulty ... is the construction of a new ideal reader who, even if he has the same academic, professional and intellectual level as the original reader, will have significantly different textual expectations and cultural knowledge. (p. 12). 
In the case of Ayeshmangal, it is not obvious that the ideal TT reader would have totally different textual expectations though, obviously, his/ her cultural and historical knowledge would not be quite same as the ideal ST reader. The historical, cultural and mythological facts presented in the ST are not expected to be familiar to the TT reader. Again, the Rangpur dialect and Dhaka-centric colloquial language are also unfamiliar to the TT reader. At the same time, though the level of linguistic competence could be quite the same for the ST and TT reader, differences will be evident in their response to many of the culture specific lexicon. James (2002), in her article points out to such issues in translation:

Indeed, the historical and cultural facts are unlikely to be known in detail along with the specific cultural situations described. Furthermore, despite considering the level of linguistic competence to be roughly equal for the ST and TT reader, certain differences may possibly be noted in response to the use of culturally specific lexis which must be considered when translating. (para. 15).

Here, the linguistic competence of readers of the ST (Ayeshamangal) and the TT (The Ballad of Ayesha) will not be very dissimilar but their cultural understanding will not be the same as pointed out by James. So, the culture specific issues and the use of myth (myth of Behula) in relation to the struggle of the protagonist will be challenging for the TT readers to apprehend clearly. These things should be considered in the translation of Ayeshamangal as this is a novel infused with Bengali culture specific issues.

\section{Translation Strategies and Procedures in The Ballad of Ayesha}

Jean-Paul Vinay and Jean Darbelnet (2004) in their essay “A Methodology for Translation” discusses seven procedures used in translation- Borrowing, Calque, Literal translation, Transposition, Modulation, Equivalence and Adaptation (pp. 128136). Now, Ayeshamangal, as stated above, seems to be intended for certain readers who are "educated, middle-class" and of Bengali origin who have some knowledge of Bangladeshi culture, history and literature. The problems of translating such a text in a foreign language like English are not simply lexical in nature, but also understanding the socio-economic, political and cultural issues. In any literary translation, historical, political and cultural references are difficult to convey in their full understanding for the TT reader. The current paper would apply some the above mentioned translation procedures to investigate how the TT, The Ballad of Ayesha attempts to transfer various issues present in the ST. This analysis, then, will help to understand the translation strategy adopted by the translator.

Again, the translator has to face issues of untranslatability, as Inam Ahmed points out- "... while translating, you may not need to put down all the things written in original text, you can easily eliminate some things which can never be explained." (Hoque, 2018, p.180). Certainly, the issue of untranslatability is a fact that the translator has to keep in mind while translating social and cultural references. So, while translating Ayeshamangal, the translator's major task has been to decide how much he should explain for the TT reader and how much he should leave for the ideal TT reader's inference. Considering these points, different cultural elements in Ayeshamangal and their translation strategy in The Ballad of Ayesha will be discussed in the following.

3.1. Cultural Categories

Newmark (1988) categorizes cultural words in five different groups: "ecology", "material culture", "social culture", "organizations, customs and ideas" and "gestures and habits". According to Newmark (1988) the category "ecology" comprises animals, plants, local winds, mountains, and plains among others (p. 96). Concepts like food, clothes, housing, transport and communications all belong to the category "material culture" (p. 97). "Social culture" refers to for example work and leisure (p. 98). Political, social, legal, religious, and artistic aspects all belong to the category "organizations, customs and ideas", referring to the institutional terms of the political and social life of a country. Also historical terms fall under that category (p. 103). As for the category "gestures and habits", it refers to how people of different cultures behave differently in certain situations, as when greeting each other with a hand shake or a kiss (p. 102). In Ayeshamangal and The Ballad of Ayesha, we can discuss examples for cultural implications in translation in some of these categories pointed out by Newmark.

\subsection{Translating Ecology}

While translating various ecological terms and words used in the ST, the translator seems to be inclined towards the method of literal translation though exceptions are found. In the first chapter, at the very beginning, we see words referring to birds- payra, chil, shokun, baaz (পায়রা, চিল, শকুন, বাজ). These source text words are translated as "pigeon", "kite", "vulture". Though baaz, the falcon is not mentioned here, it is obvious that the TT attempts literal translation here. In page 12 of the ST, we find a substantial passage in this regard where phrases like Pakhir kolotan (পাখির কলতান) is translated as “chirping of birds", bashjhare horiyaler daak (বাঁশঝাড়ে হরিয়ালের ডাক) as "green pigeon's gentle crooning in the bamboo bush", ghunpokar kutur kutur (ঘুন পোকার কুটুর কুটুর) as "the monotonous drilling of the borer insect into the wood, morog murgir kokkok (মোরগ-মুরগীর কককক) as “the chicken's cackling”. Though in the TT, in page 4, we see these translations where the translator attempts for literal transfer of words in the TT from the ST, omission of pharses like jhillir jhi jhi rob (ঝিল্লির ঝি ঝি রব) in the TT refers to the translator's inability to find out prpoper opposites in the target culture for explaining such ecological expressions. 
Again, we see words like "lajjabati pata" (লজ্জাবতী পাতা) is presented as "soft carpets of mimosa" substantiates the aforementioned attempt of literal translation, but as mentioned earlier, the translator often escapes situations he feels untranslatable as we see him shying away from translating the phrase basok gache akash mukhi phool (বাসক গাছে আকাশ মুখী ফুল) here. So, in most of the cases in translating ecological issues, the TT text uses literal translation techniques for conveying the ST words to the target text readers, but often, the translator seems to escape apparent untranslatable situations of ecological expressions of the ST.

3.3. Translating Material Culture

For Newmark (1988), "Food is for many the most sensitive and important expression of national culture; food terms are subject to the widest variety of translation procedures." (p. 97). In Ayeshamangal, different food and food items represent an important part of Bengali culture and The Ballad of Ayesha uses different strategies to translate these ST words to the TT. In the very first chapter of the ST, we find the words- "chicken fry" (চিকেন ফ্রাই), murgi-mosallam (মুরগি- মোসাল্লাম), and morog polao (মোরগ পোলাও). As chicken fry itself is an English word, it causes no problem, but in the case of other two, we see that the translator evades translating "murgi-mosallam" in the TT and uses the strategy of "Borrowing", a term that means taking a word or expression directly from another language without translation, for polao. He uses the same strategy for the words - vapa pitha (ভাপা), chitoi pitha (চিতই), puli pitha (পুলি), as he "borrows" the words vapa, chitoi, puli from the ST to the TT. The same thing has been done for the word daal (ডাল) in the ST by translating it as "daal" in TT.

Now, for the ST words daab (ডাব), pitha (পিঠা), gorom doodh (গরম দুধ) the TT uses "Adaptation", as the words are replaced with green coconut, rice cakes, milk. For the word jhalmuri (ঝালมूড়ি), we find "Description" strategy has been used, as the word is replaced with the phrase "puffed rice, mixed with bits of onion and green chili". Use of "Calque" as translation strategy can be seen for the ST word sharbat (শরবত), as it is translated as lemonade.

\subsection{Organizations, Customs and Ideas}

As Newmark (1988) says, "The political and social life of a country is reflected in its institutional terms" (p. 99), Ayeshamangal, being a novel representing history and culture of Bangaldesh uses a lot of words and expressions that fall in this category and The Ballad of Ayesha, almost all the cases translates them literally. The translator, unlike in the case of "material culture", finds it easier to translate these words as the Bengali language has naturalized most of these English words and expressions due to its colonial past. We see that many of such institutional terms are political and naturalized from English, such as -staff quarter, primary school, Red Cross, television, president are words familiar to the target culture. Then, the words - Bangladesh Biman Bahini (বাংলাদেশ বিমান বাহিনী), Chiriakhana (চিড়িয়াখানা), Bimanbandar (বিমানবন্দর), Betar Bhaban (বেতার ভবন), prodhan Samorik Ain Proshasok (প্রধান সামরিক আইন প্রশাসক), samorik tribunal (সামরিক ট্রাইব্যুনাল) are literally translated as Bangladesh Air Force, Zoo, Airport, Radio Station, Chief Martial Law Administrator, Military Tribunal. References to Sheikh Mujibur Rahman and Bangabandhu in the ST are translated literally but with "description" as we see the sentence Ora Bongobondhu ke khoon korlo kan? (অরা বঙ্গবন্ধুকে খুন করলো ক্যান?) (Hoque, 2015, p. 43) is translated- Why did they kill Bongobondhu Mujib? (Ballad 44).

For the religious words and expressions like huzur (হুজুর), auzbillah himinash shaituyaner rajim (আউযুবিল্লাহ হিমিনাশ শাইতুয়ানের রাজিম), Allah (আল্লাহ), bitarito shoytan (বিতাড়িত শয়তান), borkha (বোরখা), azan (আযান), akika (আকিকা), the TT uses literal translation strategy, "adaptation" and "borrowing" strategy. As we can see- Huzur is translated as "mullah", an adaptation; same thing is seen with the word akika as it is translated as "christen" in the TT; Allah and azan have been kept as Allah and azan; borkha has been translated as burqa. The sentence A'udhu Billahi minash shaitanir rajim (Hoque, 2018, p. 8) has been borrowed from the ST to the TT.

\section{Translating Regional Dialects: Attitude of Untranslatability}

The ST extensively uses regional dialect of Rangpur region and Dhaka-centric colloquial language is also evident in the text and the translator's attitude in this regard has been disappointing. The translator assumes an attitude of untranslatability in regard and simply uses pure English as he expresses his in ability in his discussion with Anisul Hoque when Hoque inquires about this. He says:

You've used a language that is hard to convey in English. I might write it in pure English. Like regular dialogue. I've seen you use Dhaka-centric or other colloquial language that cannot be directly transferred into English. It is difficult, and I have to decide if I should overlook it in some cases or else the reader will be at a loss. (Hoque, 2018, pp. 178-179).

Inam Ahmed, the translator, in this conversation with Mr. Hoque is evidently pointing out towards his inability or unwillingness to transfer the regional dialect of Bengali language used extensively in the ST. Mr. Ahmed is, also, expressing his indecision to frame an appropriate translation strategy in dealing with this issue. Mr. Hoque seems to be keen in keeping the taste of the colloquial language used in his novel (as every author wishes may be) when he actually inquires on this point to the translator, but Mr. Ahmed's intent to use pure English to translate these issues of the ST instead of devising a way out to keep the original suggests his lack of expertise in translating culture-specific writings. 
Now, the point is that it is indeed difficult to translate the regional dialects of Rangpur in English, but the translator's use of pure English in this regard seems to be an approach of "communicative translation" where he fails to convey the ST culture to the TT readers. As we see in the following instances:

O, Bahee. Biyao kemton khainen? (ও বাহে । বিয়াও কেমতন খাইনেন?)

Ha. Valo. (र। ভाल।)

Pet vori khaicen to? (পেট ভরি খাইছেন তো?)

Ha. (হ।) (Hoque, 2015, p. 34)

This dialogue is translated as:

'Hey, how was the feast?' Joynal yelled to the coachman.

'Yes, good.'

'Did you eat till your tummy was full?'

'Yes.' (Hoque, 2018, p. 34).

Another example is Bokul's use of Dhaka-centric colloquial language:

Bokul ese bollo, gondogol hoyche. Golaguli hoyche kailka. Toy sobai koilo cinta na korte. Gondogol thaima geche. (বকুল এসে বললো, গণ্ডোল হয়েছে। গোলাগুলি হয়েছে কাইলকা। তয় সবাই কইলো চিন্তা না করতে। গগুগোল থাইমা গেছে।) (Hoque, 2015, p. 60). This dialect of Bokul has been translated as- "Bokul returned with the same information. 'There were skirmishes in the cantonment last night. But don't worry. It's okay now." (Hoque, 2018, p. 65).

The translations here deliver the literal meanings of the words to the TT reader, but the TT reader fails to assume the beauty of the dialect and the colloquial language that is an important part of understanding the ST culture. In this case, the TT reader will fail to understand the social and cultural hierarchy evident in the dialogues between and Joynal Abedin and the gaarial (ox-cart man) in the first example and the social standing of Bokul in the second one. Now, regarding the translator's attitude of untranslatability, we can understand his position that he states but, at the same time, it is obvious that he does not attempt any strategy to overcome this problem, or at least, provide a little taste of that regional dialect to the TT readers . Surely, he could have attempted to use footnotes or endnotes not extensively but partially to portray the beauty and unique nature of those colloquial languages.

\section{Translating Mythology: Endeavoring Equivalence}

The source text Ayeshamangal uses the popular mythological story of Manasha and Behula in Bengali culture and the translation strategy of it in the TT is quite interesting. The translator uses both literal translation method and communicative translation method in this regard. Use of "Borrowing" and "Adaptation" (pp. 128-137), as discussed by Vinay and Darbelnet (2004) in "A Methodology for Translation" is evident here and in many cases the TT use "description" though the translator opts out from using footnotes. The story of Behula and Lakhinder is quite known to the target readership of the ST, but it is quite different for the TT readers. So, the translator often uses few descriptions though it does not seem adequate for the TT readers' proper understanding of the myth of Behula and its parallel connection in the ST as intended by the author. While describing Manasha, the TT uses an extra word 'the goddess'. As we see in the following example:

\section{Chand Saudagor artonad korche, amar chilo sworger baganer moto bari, oi sorbonashoni Manasha ta dhongso koreche, rajyer charidike sap ar sap, choyta putroke se hotya koreche. (চাঁদ সওদাগর আর্তনাদ করছে, আমার ছিল স্বর্গের বাগানের মত বাড়ি, অই সর্বনাশিনী মনসা তা ধ্বংস করেছে, রাজ্যের চারিদিকে সাপ আর সাপ, ছয়টা পুত্রকে সে হত্যা করেছে।) (Hoque, 2015, p. 24-25).}

In the TT, it is translated as- "“My home was like a paradise, and Manasha has turned it into a cemetery I only meet serpents in the garden. They have killed my six sons." (Hoque, 2018, p. 22). Here, we can see that the translator is opting for literal translation of the story for equivalence between the ST and the TT. Though, to avoid complexities, he also focuses on adaptation of the phrase sworger baganer moto bari by replacing it with a single English word "paradise" and the word dhongso has been replaced by using "cemetery"; this attempt of adaptation is to provide an overall equivalence by keeping the sense of the ST but fails to provide the sense of the original language adequately, as we see that "paradise" is the direct opposite of sworgo but the sense conveyed in the ST here is "a beautiful and harmonious house like the garden in paradise"; again, the translator goes for providing the sense in the case of dhongso (literally means destruction) by replacing it with "cemetery".

\section{Search for Equivalence}

The Ballad of Ayesha is mainly an attempt for free translation, though the translator often uses literal translation; thus, the approach is an in-between one. He strives for providing an overall equivalent effect in the TT in relation to the source text (ST), Ayeshamangal. Nida discusses two types of equivalence- Formal equivalence and Dynamic Equivalence where "Formal equivalence focuses attention on the message itself, in both form and content..." and "the message in the receptor language should match as closely as possible the different elements in the source language." (Munday, 2016, p. 68). This 
type of translation "allows the reader closer access to the language and customs of the source culture." (Munday, 2016, p. 68). On the other hand, Dynamic equivalence is based on 'the principle of equivalent effect' and "The message has to be tailored to the receptor's linguistic needs and cultural expectation and 'aims at complete naturalness of expression." (Munday, 2016, p. 68). Nida defines "the goal of dynamic equivalence as seeking "the closest natural equivalent to the source-language message'." (Munday, 2016, p. 68). The Ballad of Ayesha primarily focuses on this "Dynamic equivalence", as it can be seen from the translation of a joke in the ST:

Ekta chele ekta meye geche Chinese khete. Cheleta bollo, chicken fry khaba? Meyeta bollo, chickon fry. Koto chikon? Chickonta khabo na. Cheleta bollo, accha ekhon chickonta khao. Rater bela tomake motata khaoabo. (Hoque, 2015, p. 13).

The definite sexual pun used in this joke was impossible to be conveyed by a literal translation, so the translator uses a totally different joke in its place in the TT that conveys the intended sexual pun. It is as follows:

Do you know what the cocks say in the morning when they are out of the pen? They crow "What to dooooo!" And do you know what the hens reply? They say, "Fuck, fuck, fuck, fuck. (Hoque, 2018, p. 6).

The TT, in the end, becomes a combination of the translator's struggle for "Formal equivalence" and "Dynamic equivalence", though it can be termed better as what Newmark observes it as "Communicative Translation" with obvious traces of literal translation.

\section{Conclusion}

The cultural implication for translation in Ayeshamangal has been an evident one. The Ballad of Ayesha tries to find out a proper path for conveying the cultural significance of different ST issues to the TT readers without hampering the flow of the novel. Search for an equivalent effect is noticeable in the TT as for Nida "it is one of the four basic requirements of a translation, which are: i) making sense; ii) conveying the spirit and manner of the original; iii) having a natural and easy form of expression; iv) producing a similar response." (Munday, 2016, p. 68). The Ballad of Ayesha ticks almost all the boxes, but in its approach to deal with the cultural issues in the ST, it fails to convey the significance of the regional dialect of Rangpur region and the Dhaka-centric language to the TT readers which surely requires a better translation strategy and it, also, indicates the translators lack of familiarity with enough translation theories and advanced strategies. While translating a text with so much cultural issues, he should have devised a better plan to provide the taste of Bengali culture to those TL readers by using footnotes and endnotes to some extent, even though that could have hindered the flow of the translation a little. Regarding the mythological story of Behula, the TT tries to capture the spirit of the ST in this regard, but as the ideal target text reader has a cultural gap with the ideal source text reader, the intended effect of the story in parallel to the sufferings of Ayesha, the protagonist will have some significant gap in perception. Evaluation of translation method of different items of different cultural categories, discussed by Newmark (1988), like "Ecology", "Material Culture" and "Organizations, Customs and Ideas" points out to the use of literal translation method, adaptation, borrowing, calque etc. This study could not cover the issues of translating historical elements in details, so the paper would suggest further attention to this issue in future studies.

\section{References}

Coulthard, M. (1992). Linguistic constraints on translation. Studies in Translation / Estudos da Traducao, Ilha do Desterro, 28, Universidade Federal de Santa Catarina, 12.

Hoque, A. (2015). Ayeshamangal ( $4^{\text {th }}$ ed.). Pearl Publications.

Hoque, A. (2018). The ballad of Ayesha. (Traslated by Inam Ahmed). Harper Perennial, an imprint of HarperCollins.

Munday, J. (2016). Introducing translation studies. Routledge,

Newmark, P. (1988). A textbook of translation. Prentice Hall.

Toury, G. (2004). The nature and role of norms in translation, In L. Venuti (Ed), The

translation studies reader (p. 207). Routledge.

Nida, E. (2004). Principles of correspondence. In L. Venuti (Ed), The translation studies reader (pp. 157,159). Routledge.

Vinay, J. P., \& Darbelnet, J. (2004). A methodology for translation. In L. Venuti (Ed.), The translation studies reader (pp. 128-137). Routledge.

James, K. (2002). Cultural implications for translation. Translation Journal, 6(4). Retrieved 5 July 2020 from https://translationjournal.net/journal/22delight.htm 